\title{
Como Planejar e Executar buscas na Literatura Científica?
}

How to Plan and Perform Searches in the Scientific Literature?

¿Cómo Planificar y Ejecutar Búsquedas en la Literatura Científica?

\author{
Marcos Spector Azoubel ${ }^{1}$
}

[1] Pontifícia Universidade Católica de São Paulo I Título abreviado: Planejamento e Execução de Buscas Digitais I Endereço para correspondência: Rua Bela Cintra, 710, apto. 70, Consolação, São Paulo, SP. CEP: 01415-002 I Email: mazoubel@gmail.com I doi: 10.18761/PAC.2019.v10.n2.a05

Resumo: Uma breve busca na literatura permite identificar a escassez de trabalhos voltados a auxiliar pesquisadores durante a realização de suas buscas na literatura científica, o que evidencia a importância de discutir tal assunto. Os objetivos deste trabalho são discutir a importância das revisões de literatura e apresentar informações que auxiliem no planejamento e na execução de buscas eficientes na literatura científica disponível digitalmente. Para isso, são apresentadas informações gerais sobre a importância das revisões de literatura e algumas orientações para a seleção de plataformas de busca adequadas, a definição de termos de busca e a utilização de operadores booleanos, curinga, de truncagem e de proximidade. Além disso, são apresentados alguns exemplos de estudos que podem auxiliar novos pesquisadores a realizarem suas buscas na literatura. Mais importante do que seguir as orientações apresentadas é conhecer uma parte da complexidade envolvida nas buscas em literatura científica e reconhecer a necessidade de buscar informações sobre o tema e explorar as ferramentas disponibilizadas pelas diferentes plataformas de busca.

Palavras-chave: bases de dados, mecanismos de busca, metodologia, plataformas de busca, revisão de literatura.

O autor contou com bolsa de pós-doutorado (PNPD/CAPES, número 88882.315381/2019-

01). Declaro não possuir conflitos de interesse. 
Abstract: A brief literature search enables the identification of the scarcity of researches aimed at assisting researchers during their research in the scientific literature, what highlights the importance of discussing this subject. The objectives of this study are to discuss the importance of literature reviews and to provide information to assist in planning and implementation of efficient searches in the scientific literature available digitally. For this purpose, general information about the importance of literature reviews and some guidelines for the selection of suitable search platforms, the definition of search terms and the use of Boolean, wildcard, truncation and proximity operators are presented. In addition, some examples of studies that may help new researchers to search the literature are presented. More important than following the guidelines presented here is to have contact with a part of the complexity involved in the searches in scientific literature and to recognize the need for seeking information about this subject and to explore the tools offered by diverse search platforms.

Keywords: database, literature review, methodology, scientific research, search platforms, search engines.

Resumen: Una búsqueda breve en la literatura permite identificar la escasez de trabajos con objetivo de auxiliar la realización de su búsqueda en la literatura científica, lo que evidencia la importancia de tratar este tema. Los objetivos de este trabajo son discutir la importancia de las revisiones de literatura y presentar informaciones que ayuden en la planificación y la ejecución de búsquedas eficientes en la literatura científica disponible digitalmente. Para ello, se presentan informaciones generales sobre la importancia de las revisiones de literatura y algunas orientaciones para la selección de plataformas de búsqueda adecuadas, la definición de términos de búsqueda y la utilización de operadores booleanos, comodín, de truncamiento y de proximidad. Además, se presentan algunos ejemplos de estudios que pueden auxiliar a nuevos investigadores a realizar sus búsquedas en la literatura. Más importante que seguir las directrices que se presentan es conocer una parte de la complejidad implicada en las búsquedas de la literatura científica y reconocer la necesidad de buscar información sobre el tema y explorar las herramientas proporcionadas por las diferentes plataformas de búsqueda.

Palabras clave: bases de datos, buscadores, metodología, plataformas de búsqueda, revisión de literatura. 
Na obra de Skinner $(1953 ; 1957 ; 1974)$ é recorrente o argumento de que o cientista tem como objetivo descrever leis de funcionamento da natureza, descrições de contingências, que permitam ações efetivas sobre as contingências descritas. Tais leis sobre o funcionamento do mundo devem não apenas descrever relações entre eventos do passado, mas possibilitar previsão e controle no futuro (Skinner, 1953), além de auxiliar na interpretação de eventos cotidianos (Holland \& Skinner, 1961; Skinner, 1974).

Em sua concepção, o conhecimento científico não possui qualquer valor quando isolado. A ciência não é apenas o fazer individual dos cientistas e as práticas da comunidade verbal dos mesmos, que selecionam e mantêm seus comportamentos, mas também seus produtos verbais (Skinner, 1957). Contribui para o sucesso do empreendimento científico o fato de que as ações dos cientistas muitas vezes produzem estímulos textuais, permitindo alcançar potenciais beneficiários dos seus conhecimentos, por meio de artigos científicos, livros, teoremas, conceitos etc. Dessa maneira, a comunicação científica é parte inseparável do fazer ciência.

A valorização da comunicação científica, especialmente por meio de artigos científicos, o advento da internet e o crescimento da produção acadêmica mundial criaram uma situação em que, ao mesmo tempo em que nunca se produziu tanta informação, nunca foi tão difícil acompanhar o desenvolvimento das diferentes áreas de produção de conhecimento e selecionar os textos relevantes para objetivos específicos, avaliá-los criticamente e compreender o estágio de desenvolvimento de determinada área (Garvey, 2014). Nesse contexto, destaca-se a importância das revisões de literatura como forma de lidar com a variedade de publicações existentes. Existem diversas definições de revisão de literatura (cf. Bogopane, 2013), mas, numa definição simples, a revisão de literatura pode ser entendida como um processo que envolve necessariamente (1) buscas na literatura científica, feitas com base num tema de interesse, e (2) seleção e avaliação crítica de parte da literatura encontrada.

Os objetivos deste trabalho são discutir a importância das revisões de literatura e apresentar informações que auxiliem no planejamento e na execução de buscas eficientes na literatura científica disponível digitalmente. Dessa maneira, não será discutido aqui como realizar a segunda etapa das revisões de literatura: a seleção e a avaliação de partes da literatura. Isso porque existe grande variedade de critérios para seleção dos textos e para o estabelecimento de categorias de análise, a depender dos objetivos, inviabilizando a discussão adequada de todas as etapas em apenas um texto.

Uma breve busca na literatura da área permite identificar a escassez de trabalhos voltados a auxiliar pesquisadores durante a realização de suas buscas na literatura científica, o que evidencia a importância de discutir tal assunto. É possível identificar trabalhos tratando de plataformas de busca específicas (e.g., Gómez-Sánchez, 2016; Sánchez, Gómez \& Gallardo, 2016) e descrevendo as classificações de tipos de revisão de literatura (e.g., Souza, Silva \& Carvalho, 2010; Mendes, Silveira \& Galvão, 2008), mas é possível verificar carência de trabalhos voltados a auxiliar pesquisadores a selecionar termos de busca e plataformas de busca e definir seus procedimentos de busca.

\section{Por que fazer revisões de literatura?}

Qualquer pesquisa acadêmica costuma surgir de uma pergunta sobre o mundo (Runkel \& McGrath, 1972), mesmo se colocada de maneira ainda imprecisa (Luna, 1996/2009). Por exemplo, um analista do comportamento em formação que frequenta eventos da área pode se dar conta do pequeno número de fluminenses presentes em tais eventos e perguntar-se: "Por que será que existem poucos analistas do comportamento do Rio de Janeiro?". Caso ele leve esta pergunta para seu orientador do trabalho de conclusão de curso, como sugestão de tema de pesquisa, deve escutar o pedido do seu orientador para que faça uma busca na literatura cientifica.

Afinal, a revisão de literatura constitui o primeiro passo para a construção do conhecimento científico em qualquer linha de pesquisa e serve para identificar o que já foi estudado sobre este tema, se é que algo já estudado neste tema ou em temas próximos a ele, quais métodos foram empregados nestes estudos e possíveis lacunas e oportunidades para o surgimento de novas pesquisas. Com base no exemplo apresen- 
tado, ele poderia notar, por exemplo, que estudos historiográficos foram realizados, examinando a história da disseminação da análise do comportamento no Brasil, mas que há poucos estudos abordando como a análise do comportamento chegou ao Rio de Janeiro. A partir daí, ele poderia, dentre várias possibilidades, decidir realizar um estudo com objetivo de identificar quais foram os primeiros cursos de ensino superior que abordaram assuntos da análise do comportamento no estado, analisando documentos desses cursos, quais conteúdos foram abordados e quais foram os professores responsáveis. Como visto, uma revisão da literatura deve permitir que o pesquisador encontre lacunas a serem preenchidas no conhecimento produzido sobre determinado assunto e métodos de pesquisa adequados para o estudo desse assunto.

$\mathrm{Na}$ discussão dos resultados de qualquer pesquisa, para responder ao problema de pesquisa ${ }^{1}$, os achados da revisão de literatura voltam a ser necessários. Nesta etapa, os dados produzidos na pesquisa devem ser articulados com os dados presentes na literatura. Continuando no exemplo hipotético, vamos imaginar que o autor da pesquisa tenha lido, como parte da sua revisão de literatura inicial, que os primeiros cursos ensinando conteúdos de análise do comportamento no Rio de Janeiro tenham sido ministrados por professores sem formação específica nesta teoria e, a partir dos seus dados, tenha encontrado informações que corroboram com a hipótese presente na literatura. Caberia apresentar a articulação entre os seus achados e os achados previamente encontrados na literatura.

Num segundo exemplo hipotético, um aluno de mestrado, a partir do seu interesse pessoal em ativismo e ação social, após uma revisão inicial da literatura que permitiu encontrar uma diversidade de estudos de analistas do comportamento sobre o tema, pode colocar-se o seguinte problema de pesquisa: quais são as principais características das pesquisas de analistas do comportamento que lidam com ação social?

O problema de pesquisa deve encaminhar o pesquisador ao método de pesquisa mais adequado, que provenha informações capazes de auxiliar a re-

1 Problemas de pesquisa são constituídos por uma pergunta ou por um conjunto de perguntas que destacam as informações que a pesquisa buscará produzir (cf. Luna, 1996/2009). sponder o problema de pesquisa adequadamente. É possível que, para o nosso mestrando hipotético, o melhor caminho seja analisar os artigos publicados em periódicos importantes da área, a fim de identificar como os analistas do comportamento investigaram temas relacionados à ação social.

Nessa situação, estamos falando de uma pesquisa de revisão crítica da literatura. Em outras palavras, o método selecionado para responder ao problema de pesquisa e chegar a conclusões sobre o mundo é uma revisão de literatura. Pesquisas do tipo podem servir a variados objetivos, porém, de maneira geral, podem auxiliar a: apontar e discutir possíveis soluções para problemas similares; identificar avanços, retrocessos ou tendências de publicação na área; examinar métodos utilizados para lidar com determinada questão; avaliar tendências teóricas de determinada área.

Enfim, a revisão de literatura é uma etapa necessária para realização de qualquer pesquisa acadêmica e, nos casos das pesquisas de revisão crítica da literatura, compreende também um conjunto de procedimentos realizados para coletar informações e analisar dados a fim de responder a um problema de pesquisa proposto. Para ambos casos, as buscas na literatura científica são requisitos.

\section{Como planejar e executar buscas na literatura científica?}

De acordo com Rumph e Runkel (1972), para se fazer uma pesquisa, parte-se de uma questão (ou algumas questões) e então uma série de decisões interdependentes deve ser tomada com intuito de chegar a conclusões acerca dessa questão. Os passos tomados a fim de responder as questões colocadas inicialmente costumam ser desviados, podendo levar o pesquisador a refazer etapas anteriores e modificar os caminhos constantemente. O processo de pesquisa trata-se, normalmente, de um ir e vir.

Da mesma maneira, o processo de realizar buscas na literatura científica é composto por passos interdependentes, que podem sofrer diversos desvios. O esquema apresentado na Figura 1 exemplifica uma direção comum em buscas na literatura científica. Na prática, a ordem pode ser diferente, alguns passos podem não ser seguidos e outros passos podem ser adicionados. 


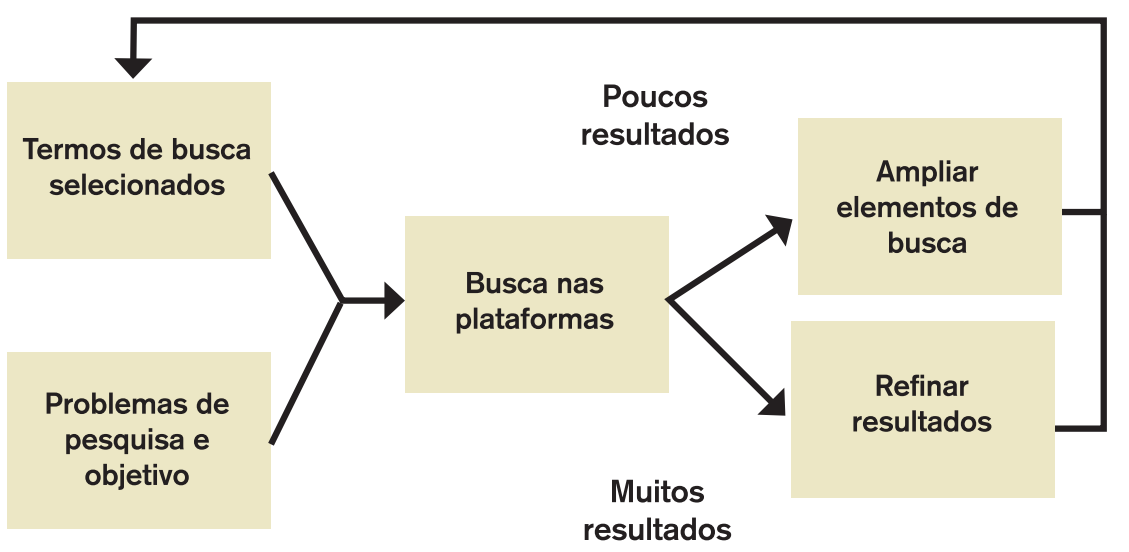

Figura 1. Esquema que exemplifica o ir e vir do processo de buscar na literatura científica.

É comum que o pesquisador já tenha lido algo acerca do tema sobre o qual pesquisará e que tenha uma questão mais ou menos definida, mesmo que seja ainda uma intenção imprecisa (cf. Luna, 1996/2009). Por exemplo, um estudante poderia ter interesse em pesquisar algo sobre esquizofrenia e análise do comportamento. Ele poderia usar "esquizofrenia" e "análise do comportamento" ou as palavras-chave dos trabalhos que já tenha lido sobre o tema, se este for o caso, como termos de busca e poderia usar as plataformas de busca que conhecesse, como o Google Acadêmico. Caso ele encontrasse um número insatisfatório de trabalhos usando este procedimento, ele poderia ampliar os elementos de busca, variando os termos empregados, podendo usar termos menos específicos, e adicionando novas plataformas de busca. Caso encontrasse um número muito grande de trabalhos, ele poderia refinar os seus procedimentos de busca, delimitando um período específico, utilizando termos mais específicos, selecionando apenas um tipo de publicação (e.g., artigos publicados em periódicos, teses, dissertações ou livros). Enfim, é comum que os passos precisem ser feitos e refeitos diversas vezes até que se chegue a um número adequado de trabalhos que auxiliem a responder a questão colocada antes de começar as buscas.

Feitas essas colocações sobre o ir e vir das buscas na literatura científica, a seguir serão apresentadas algumas indicações para auxiliar o leitor a selecionar plataformas de busca, definir termos de busca e utilizar alguns elementos que podem facilitar a realização de buscas avançadas. A ordem de apresentação dos assuntos não representa, necessariamente, a ordem que as buscas devem seguir.

\section{Seleção das plataformas de busca}

Optou-se aqui pela utilização da expressão "plataformas de busca", em vez de "bases de dados", pois serão abordados aqui alguns portais eletrônicos que se caracterizam como bibliotecas digitais (e.g., Periódicos CAPES) e outras que fornecem ferramentas de busca que acessam diversas bases de dados (e.g., PsycNET). Assim, parece mais adequado utilizar um termo mais amplo, plataformas de busca, do que o termo mais restrito, bases de dados, para minimizar possíveis confusões.

Bases de dados científicas são conjuntos de registros bibliográficos que são buscados e processados por meio de uma ferramenta eletrônica, no caso das bases digitais, ou por algum suporte físico, no caso das bases de dados não digitais (Linares, Herrera \& Alfaro, 2016). Sampaio e Serradas (2009) categorizaram as bases de dados que dão acesso à literatura científica em referenciais, que apresentam apenas as referências dos trabalhos e, normalmente, o endereço eletrônico para acessar o texto completo; de textos completos, que indexam os trabalhos completos em seus bancos de dados; e de índices de citação, que apresentam, além das referências e/ou os trabalhos completos, um exame das citações dos trabalhos presentes na base de dados.

Uma seleção de plataformas de busca que permitem acesso a trabalhos da Psicologia e de áreas afins está disponível na Tabela 1. Importante esclarecer que existem diversas outras plataformas de busca, de maneira que cabe ao pesquisador interessado em revisar a literatura explorar outras possibilidades existentes (e.g., ERIC, Scopus, Springer, Web of Science e Wiley). 


\section{Tabela 1 Títulos, informações gerais e endereços eletrônicos de uma seleção de plataformas de busca que} permitem acesso a trabalhos da Psicologia e de áreas afins.

\begin{abstract}
Título da plataforma Informações gerais sobre a plataforma de busca de busca

Biblioteca Digital de

Teses e Dissertações

Catálogo de Teses e Dissertações da CAPES

Google Acadêmico

A Biblioteca Digital de Teses e Dissertações é uma biblioteca digital que indexa trabalhos completos defendidos em todo o Brasil e por brasileiros no exterior. Estão disponíveis cerca de 125 mil teses e 340 mil dissertações oriundas de 105 Instituições (e.g., UFScar, USP, UnB, UEL, PUC-SP etc.).

O Catálogo de Teses e Dissertações da CAPES indexa os resumos das teses e dissertações defendidas nos programas de pós-graduação stricto sensu no Brasil desde 1987. Estas informações podem ser acessadas por meio do site da Catálogo de Teses e Dissertações da CAPES ou do Periódicos CAPES.

O Google Acadêmico é a plataforma de buscas em literatura científica do Google. Apesar de dar acesso a grande número de trabalhos acadêmicos, não estão públicas as informações sobre bases de dados, periódicos e artigos acessados pela plataforma. Cabe destacar que esta plataforma busca também por textos da chamada "literatura cinza": não avaliados por pares, não acadêmicos e, por vezes, de qualidade duvidosa.
\end{abstract}

Index Psi

PePSIC

Periódicos CAPES

PsycNET

PubMed

Science Direct

O Index Psi é composto por quatro bases de dados em Psicologia e áreas afins: Index Psi Periódicos Técnico-Científicos, que indexa artigos publicados em periódicos científicos de Psicologia, Index Psi Divulgação Científica, que indexa materiais de divulgação científi$\mathrm{ca}$, Index Psi TESES, que indexa teses e dissertações e Index Psi LIVROS, que agrupa livros.

O PePSIC é um portal de periódicos científicos em Psicologia e áreas afins que publica periódicos de 11 países latino-americanos.

O Periódicos CAPES é considerado a maior biblioteca digital do mundo. Seu acervo conta com mais de 38 mil títulos com texto completo, 134 bases referenciais, 11 bases dedicadas exclusivamente a patentes, além de livros, enciclopédias e obras de referência, normas técnicas, estatísticas e conteúdo audiovisual. Inclui algumas das bases de dados mais importantes da Psicologia e da Educação, como PsycINFO, ERIC, BVS-Psi etc.

O PsycNET é a plataforma de busca da American Psychological Association (APA) que engloba seis bases de dados com literatura da Psicologia e das Ciências Sociais. Dentre estas bases, destaca-se a PsycINFO, que cobre artigos científicos da psicologia publicados em periódicos, livros e teses datadas de 1597 até o presente. É considerada a mais ampla base de dados para trabalhos de Psicologia do mundo.

O PubMed é um serviço desenvolvido e mantido pelo Centro Nacional de Informação Biotecnológica (NCBI) dos EUA. Possui duas bases de dados com livros e artigos científicos de áreas da biomedicina e da saúde: PubMed Central e PubMed. O PubMed Central é uma base que contém apenas artigos digitais gratuitos. Estão disponíveis mais de 3 milhões de artigos completos. O PubMed é uma base referencial que contém resumos, títulos e informações sobre textos científicos.

O Science Direct é a plataforma de busca da Elsevier, considerada a maior editora de literatura científica do mundo, publica mais de 2500 periódicos, de várias partes do mundo.

\section{Endereço eletrônico da} plataforma de busca

http://bdtd.ibict.br/

http://catalogodeteses.capes. gov.br/catalogo-teses/\#!/

https://scholar.google.com.br/

http://newpsi.bvs-psi.org $\mathrm{br} / \mathrm{cgi}$-bin/wxis1660.exe/ iah/?lsisScript=iah/iah. xis\&lang=P\&base=INDEXPSI

http://portal.pepsic.bvsalud.org/ php/index.php?lang=pt

http://www.periodicos.capes. gov.br/

http://psycnet.apa.org/

https://www.ncbi.nlm.nih.gov/

http://www.periodicos.capes. gov.br/ 
Para definir quais plataformas de busca devem ser utilizadas é preciso considerar a abrangência das mesmas. Por exemplo, caso seja objetivo fazer uma revisão ampla da literatura, é recomendado utilizar as plataformas que dão acesso às bases de dados mais extensas; caso o problema de pesquisa envolva temas estudados por outras disciplinas científicas, é importante considerar o acesso a plataformas que conectam-se a bases de dados dessas disciplinas. Cabe ainda examinar, ao selecionar as plataformas de busca, se existem redundâncias: algumas plataformas dão acesso aos trabalhos de bases de dados semelhantes. Este é o caso do Periódicos CAPES e do PsycINFO, o primeiro dá acesso ao segundo, de forma que, a princípio, fazer a busca em ambos resultaria em trabalho desnecessário.

Outro aspecto que deve ser considerado é a possibilidade de realização de buscas avançadas e de refinamentos dos resultados das buscas. A título de exemplo, o Science Direct permite a realização de buscas avançadas com utilização de diversos recursos para a realização de buscas avançadas (i.e., operadores booleanos, de truncagem e de proximidade), enquanto o Google Acadêmico não permite a mesma complexidade nas suas buscas.

\section{Definição de termos de busca}

Neste tópico é importante começar esclarecendo as diferenças entre palavras-chave e descritores. De acordo com Sabadini, Sampaio e Koller (2009), costuma haver confusão na utilização desses termos. As palavras-chave são termos selecionados pelo(s) autor(es) do artigo. Os critérios para escolher tais termos são variados. A diversidade de palavraschave pode dificultar a realização de buscas na literatura, de forma que a comunidade científica elaborou conjuntos de descritores (Castro, 2001). De forma resumida, os descritores, também chamados de termos controlados ou vocabulário controlado, são termos selecionados por especialistas e utilizados por certas bases de dados ao indexar artigos científicos.

Dentre os conjuntos de descritores existentes, podemos destacar alguns com importância reconhecida para a Psicologia: o Descritores em Ciências da Saúde (DeCS), utilizado para indexação de trabalhos na Biblioteca Virtual em Saúde (BVS); o
Medical Subject Headings (MeSH), que serve para a indexação de artigos na base de dados da Medline; o Terminologia em Psicologia, usado ao indexar trabalhos acadêmicos no Index Psi; o APA Thesaurus, aplicado para a inserção de textos das bases de dados da American Psychological Association (APA).

Os termos de busca selecionados podem incluir descritores ou não, a depender da pertinência em cada situação. Se a busca está sendo realizada a fim de encontrar trabalhos de uma linha de pesquisa pouco popular ou bastante recente, é possível que não tenham sido criados descritores para trabalhos sobre esse tema de pesquisa. Por outro lado, quando há grande variação nos termos utilizados em determinada área de produção de conhecimentos, pode ser especialmente relevante utilizar descritores. Em qualquer dos casos, cabe ao menos um exame dos descritores existentes para avaliar a pertinência ou não de utilizá-los como termos de busca.

\section{Utilização de operadores booleanos, curinga, de truncagem e de proximidade}

Operadores booleanos são palavras que estabelecem diferentes relações lógicas entre termos de busca utilizados (Sánchez, Gómez \& Gallardo, 2016). São eles AND, OR e OR NOT. De modo geral, elas informam para a plataforma como deve ser feita a combinação entre os termos buscados. Podem haver pequenas variações, por exemplo, em algumas plataformas de buscas, o operador "OR NOT" é trocado por apenas "NOT". Por isso, é necessário avaliar o funcionamento desses operadores em cada plataforma.

Um exemplo das relações lógicas estabelecidas entre os termos de busca, por meio diferentes operadores booleanos, pode ser visualizado no esquema apresentado na Figura 2. Um pesquisador que estivesse interessado em encontrar trabalhos tanto sobre behaviorismo quanto sobre pragmatismo poderia utilizar, entre estes termos de busca, o operador booleano "OR" e, assim, encontrar trabalhos em que qualquer um (ou ambos) dos termos estivesse presente. Caso o pesquisador quisesse encontrar trabalhos que discutissem sobre behaviorismo e pragmatismo, poderia ser empregado o operador "AND", de forma que resultariam apenas trabalhos em que os dois termos aparecessem. Por 
fim, se o pesquisador se interessasse sobre trabalhos que tratassem apenas sobre behaviorismo e que não abordassem o pragmatismo, ele poderia usar o operador "AND NOT", recuperando trabalhos que contivessem o termo behaviorismo e necessariamente não contivessem o termo pragmatismo.

É comum que as plataformas de busca, em suas opções de buscas simples, ofereçam esses operadores em caixas de seleção, ao lado dos campos para inserção dos termos de busca. Nas opções de buscas avançadas é frequente que os operadores booleanos tenham que ser digitados manualmente.
Outro recurso para aprimorar a busca é o operador curinga, que trata-se de um elemento que é substituível por qualquer outro caractere. Usualmente, este operador é representado pelo ponto de interrogação (“?”). Alguém interessado em procedimentos de ensino para alunos com dificuldades de aprendizagem poderia utilizar o termo de busca "alun?s", recuperando então trabalhos que possuam o termo alunos e alunas. Da mesma forma que ocorre com os operadores booleanos, pode haver variações na forma do operador curinga.
Behaviorismo OR Pragmatismo

Recupera trabalhos com um dos termos "behaviorismo" e "pragmatismo" ou com ambos.

Behaviorismo AND Pragmatismo Recupera trabalhos com dois termos "behaviorismo" e "pragmatismo".

Behaviorismo AND NOT Pragmatismo Recupera trabalhos com o termo "behaviorismo", mas que não tenham o termo "pragmatismo".
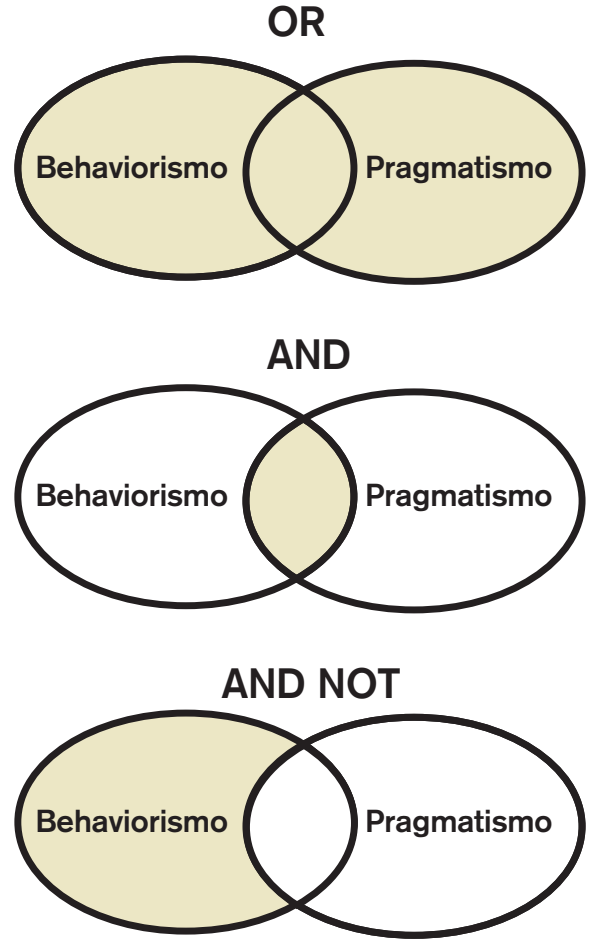

Figura 2. À esquerda, campos de buscas hipotéticos com diferentes operados booleanos, a partir dos termos "behaviorismo" e "pragmatismo" e descrição dos resultados recuperados; à direita, representação visual, por meio de conjuntos, dos resultados dessas buscas.

Recorrentemente o asterisco $\left(^{*}\right)$ é utilizado nas plataformas de busca como operador de truncagem. Este elemento amplia a busca, partindo de um prefixo, sufixo ou ambos, adicionando quaisquer elementos para preenchimento dos termos. Como exemplo, um interessado em questões relativas às neurociências poderia utilizar o prefixo neuro seguido do operador de truncagem. O uso do termo de busca neuro* ${ }^{*}$ recuperaria trabalhos contendo quaisquer palavras que começassem com neuro e terminassem de qualquer maneira, incluindo neurologia, neuroanatomia, neuropsicologia etc. Em outro caso, alguém que utilizasse o termo de busca *behaviorismo recuperaria trabalhos contendo palavras neobehaviorismo. Por fim, também é possível usar o operador de truncagem no começo e no final de um termo: alguém que empregasse o termo de busca ${ }^{\star}$ psico $^{\star}$ recuperaria trabalhos con- 
tendo as palavras neuropsicologia, biopsicossocial, exopsicologia etc.

As aspas e a chave são elementos que costumam, a depender de cada plataforma de busca, ser utilizados para limitar a busca apenas aos termos que estiverem adjacentes no texto, funcionando como operadores de proximidade. Por exemplo, um estudante que quisesse buscar textos sobre análise do comportamento e utilizasse essa expressão sem esses operadores de proximidade encontraria grande variedade de textos que contivessem os termos mesmo se separados, provavelmente recuperando diversos textos sobre outros temas. Por sua vez, "análise do comportamento" ou \{análise do comportamento\} mostrará apenas os resultados que contiverem essas três palavras juntas e nesta ordem, sem nenhuma outra palavra entre elas.

Pode haver variações na forma de utilização desses operadores entre as diferentes plataformas de busca, bem como alguns operadores não funcionam em todas as bases. Dessa forma, é primordial que o pesquisador verifique as possibilidades disponíveis em cada plataforma de busca selecionada.

A utilização desses operadores, bem como a seleção das plataformas de busca e dos termos de pesquisa são decisões interdependentes que devem ser tomadas a depender dos objetivos do pesquisador. Para ilustrar como essas decisões costumam ser tomadas, subordinadas a uma questão a ser investigada, um exemplo de pesquisa de revisão de literatura será examinado.

\section{Exame de um exemplo de pesquisa de revisão de literatura}

Devido à já mencionada escassez de materiais que auxiliem pesquisadores a empreenderem as suas buscas na literatura científica, resta recorrer a exemplos de trabalhos que tenham descrito adequadamente os critérios para seleção de termos, plataformas e procedimentos de busca. Aqui será tomado o exemplo da dissertação de Abbud (2016), intitulada "Orientação de pais como estratégia de prevenção de problemas de comportamento infantis: revisão da literatura e proposta de intervenção segundo princípios da análise do comportamento". As seguintes perguntas nortearam o seu trabalho:
Quais são as características dos programas de prevenção primária de problemas de comportamento infantis, do período de 2004 a 2014, que utilizaram como estratégia a capacitação de cuidadores?

Quais são as características críticas para a efetividade de um programa de prevenção de problemas de comportamento infantis, segundo princípios da Análise do Comportamento? (Abbud, 2016, p. 24)

Para responder essas perguntas a autora fez uma revisão da literatura indexada às bases de dados PsycINFO e Science Direct. Os critérios para seleção dessas bases de dados foram a abrangência das mesmas, que reúnem grande número de periódicos de diversas nacionalidades, e o fato de elas agruparem trabalhos de Psicologia, entre outras áreas de produção de conhecimento.

Já para a seleção dos termos de busca, a autora (Abbud, 2016) pesquisou por descritores presentes no DeCS e no APA Thesaurus. Nas plataformas de busca de ambos vocabulários controlados, a pesquisadora utilizou os termos "behavior", "prevent", "parent" e "family" para recuperar descritores relacionados à prevenção de problemas de comportamento. Após essa busca, foram selecionados 34 descritores, para serem utilizados como termos de busca, relacionados a esses termos buscados.

Tais termos de busca foram divididos em grupos: (a) 13 descritores relacionados ao comportamento; (b) 15 descritores relacionados à família e (c) 6 descritores relacionados à prevenção. Posteriormente, o procedimento de busca foi realizado de forma que todos os trabalhos recuperados contivessem ao menos um termos relacionado a cada grupo mencionado ( $a, b$ e c). Isto é, para ser selecionado na busca, o trabalho precisaria abordar, simultaneamente, os temas comportamento, família e prevenção. Devido ao número reduzido de descritores relacionados à prevenção, aspecto central do trabalho, a autora optou por utilizar o operador de truncagem, realizando as buscas com o termo "preven*", de forma a encontrar trabalhos que possuam quaisquer palavras começadas com o prefixo "preven" e terminadas de qualquer maneira (e.g., prevenção, prevenir, prevention e preventive). 
Para que todos os trabalhos contivessem, ao menos, um termo relacionado a cada grupo temático ( $\mathrm{a}, \mathrm{b}$ e c), os procedimentos de busca seguiram esta lógica: (\{Child Behavior\} OR \{Child Behavior Disorders\} OR \{Maternal Behavior\} OR \{Paternal Behavior\} OR \{Risk reduction Behavior\} OR \{Antisocial Personality Disorder\} OR \{Social Behavior Disorders\} OR \{Attention Deficit and Disruptive Behavior Disorders $\}$ OR \{Aggressive Behavior $\}$ ) AND (\{Parent Child Communication\} OR \{Parent Child Relations OR \{Parent Educational Background\} OR \{Parent Training OR \{Parental Attitudes\} OR \{Parenting Skills\} OR \{Parenting Style\} OR \{Family Practice\} OR \{Family Relations $\}$ OR \{Family Conflict $\}$ OR \{Family Intervention\} OR \{Family Life Education OR \{Family Relation\} OR \{Family Therapy OR \{Family Work Relationship\}) AND (Preven* OR \{Early Intervention\}).

Como é possível notar, foram utilizados operadores de proximidade $(\{\})$, operadores booleanos (OR e AND) e o operador de truncagem $\left(^{*}\right)$. Os grupos temáticos de termos foram colocados entre parênteses e seguidos do operador AND para que fossem recuperados trabalhos com ao menos um termo de cada grupo, tendo sido utilizado OR entre os termos de cada grupo para que qualquer um deles pudesse estar presentes.

Por fim, espera-se que o exemplo sirva como um modelo que auxilie novas buscas na literatura científica. Cabe relembrar que os procedimentos de busca dependem da pergunta que foi colocada. Algumas perguntas demandarão procedimentos de busca mais simples do que o exemplo analisado, enquanto certas perguntas requererão procedimentos ainda mais complexos do que esse modelo. Cada caso deve ser avaliado individualmente.

\section{Considerações Finais}

As indicações apresentadas aqui não devem ser entendidas como regras estritas. O ir e vir das buscas em literatura científica costuma demandar constantes reflexões e revisões dos passos tomados. Isso é especialmente importante num contexto em que mudanças no funcionamento das plataformas de busca não são incomuns e que há variações nos funciona- mentos dessas plataformas. Assim, mais importante do que seguir as orientações apresentadas até aqui é conhecer uma parte da complexidade envolvida nas buscas em literatura científica e reconhecer a necessidade de buscar informações sobre o tema e explorar ao máximo as ferramentas disponibilizadas pelas diferentes plataformas de busca.

Dentre as diversas possibilidades de aprimoramento encontram-se os cursos gratuitos oferecidos no endereço eletrônico do Periódicos CAPES (http://periodicos.capes.gov.br), o contato com bibliotecários da área, a leitura dos tutoriais que a maior parte das plataformas de busca oferece e o exame dos Métodos de trabalhos acadêmicos que descreveram detalhadamente seus passos na busca de literatura científica e seus critérios para seleção de plataformas de busca, de termos de busca e de procedimentos de busca (e.g., Abbud, 2016; Bandeira, 2017; Leonardi, 2016). É importante que os pesquisadores explorem essas iniciativas para que possam encontrar modelos adequados a serem seguidos.

Por fim, o presente trabalho ateve-se à discussão sobre procedimentos para o planejamento e a execução de buscas eficientes na literatura, de forma que tópicos importantes para a realização de pesquisas de revisão de literatura não foram abordados. Pode ser relevante, para os pesquisadores interessados no tema, consultar as recomendações PRISMA (cf. Galvão, Pansani \& Harrad, 2015), que apresentam elementos para descrição adequada de pesquisas de revisão sistemática da literatura, o livro de Luna (1996/2009) sobre planejamento de pesquisa, que inclui discussões sobre tratamento de dados, e o livro de Laurenti, Lopes e Araujo (2016), que aborda aspectos metodológicos da pesquisa teórica na Psicologia.

\section{Referências}

Abbud, G. (2016). Orientação de pais como estratégia de prevenção de problemas de comportamento infantis: revisão da literatura e proposta de intervenção segundo princípios da análise do comportamento. (Dissertação de Mestrado). Pontifícia Universidade de São Paulo, São Paulo, SP.

Bandeira, V. G. (2017). Intervenções analítico-comportamentais para a esquizofrenia: uma 
revisão sistemática da literatura. (Dissertação de Mestrado). Pontifícia Universidade de São Paulo, São Paulo, SP.

Bogopane, L. P. (2013). A critical review of pertinent qualitative research processes, approaches, and tools in social sciences. Journal of Social Sciences, 35(3), 217-229. https://doi.org/10.108 $0 / 09718923.2013 .11893161$

Castro, E. (2001). Terminologia, palavras-chave, descritores em saúde: qual a sua utilidade?. Jornal Brasileiro de Aids, 2(1), 51-61.

Eyre-Walker, A. \& Stoletzki, N. (2013). The Assessment of Science: The Relative Merits of Post-Publication Review, the Impact Factor, and the Number of Citations. PLOS Biology, 11(10), 1-8. http://doi.org/10.1371/journal. pbio. 1001675

Fraley, R. C., \& Vazire, S. (2014). The N-pact factor: Evaluating the quality of empirical journals with respect to sample size and statistical power. PloS one, 9(10), e109019. https://doi. org/10.1371/journal.pone.0109019

Galvão, T. F., Pansani, T. S. A. \& Harrad, D. (2015). Principais itens para relatar Revisões sistemáticas e Meta-análises: a recomendação PRISMA. Epidemiologia e Serviços de Saúde, 24(2), 335-342. https://dx.doi.org/10.5123/ S167949742015000200017

Garvey, W. D. (2014). Communication: the essence of science. New York, NY: Pergamon Press.

Laurenti, C., Lopes, C. E., \& Araujo, S. F. (Eds.). (2016). Pesquisa teórica em psicologia: aspectos filosóficos e metodológicos. São Paulo: Hogrefe.

Leonardi, J. L. (2016). Prática baseada em evidências em psicologia e a eficácia da análise do comportamento clínica. (Tese de Doutorado). Universidade de São Paulo, SP.

Linares, R., Herrera, J. \& Alfaro, L. (2016) AliciaVR: Exploration of scientific articles in an immersive virtual environment with natural user interfaces. IEEE Ecuador Technical Chapters Meeting, 1-6. https://doi.org/10.1109/ ETCM.2016.7750829

Luna, S. V. (2009). Planejamento de pesquisa: uma introdução. São Paulo: EDUC. (Original publicado em 1996)

Mendes, K. D. S., Silveira, R. C. D. C. P., \& Galvão, C. M. (2008). Revisão integrativa: método de pesquisa para a incorporação de evidências na saúde e na enfermagem. Texto \& ContextoEnfermagem, 17(4), 758-764. https://doi. org/10.1590/S0104-07072008000400018

Runkel, P. J. \& MacGrath, J. E. (1972). Research on human behavior. New York: Holt, Rinehart \& Winston.

Sabadini, A. A. Z. P., Sampaio, M. I. C. \& Nascimento, M. M. (2009). Preparando um periódico científico. In A. A. Z. P. Sabadini, M. I. C. Sampaio \& S. H. Koller (Eds.). Publicar em psicologia: um enfoque para a revista científica (pp. 35-76). São Paulo: Associação Brasileira de Editores Científicos de Psicologia.

Sampaio, M. I. C. \& Serradas, A. (2009). O movimento de acesso aberto, os repositórios e as revistas científicas. In A. Z. P. Sabadini, M. I. C. Sampaio \& S. H. Koller (Eds.), Publicar em Psicologia: um enfoque para a revista científica (pp. 75- 86). São Paulo: Associação Brasileira de Editores Científicos de Psicologia; Instituto de Psicologia da Universidade de São Paulo.

Sánchez, A. F. G., Gómez, C. E., \& Gallardo, P. S. (2016). MEDLINE-PubMed: la puerta de acceso al conocimento en Ciencias de la Salud. Metas de enfermería, 19(5), 49-53.

Skinner, B. F. (1953). Science and human behavior. New York: Macmillan.

Skinner, B. F. (1957). Verbal behavior. New York: Appleton-Century-Crofts. https://doi. org/10.1037/11256-000

Skinner, B. F. (1971). Beyond freedom and dignity. Indianapolis, FL: Hackett Publishing Company. Skinner, B. F. (1974). About behaviorism. New York: Alfred A. Knopf.

Souza, M. T. de, Silva, M. D. \& Carvalho, R. (2009). Revisão integrativa: o que é e como fazer. Einstein, 8(1), 102-106. https://doi.org/10.1590/ s1679-45082010rw1134

\section{Informações do Artigo}

\section{Histórico do artigo:}

submetido em: 25/03/2019

primeira decisão editorial: 14/12/2019

aceito em: 19/12/2019 